\title{
Comparison of detection techniques in optical CDMA access network for point to multipoint configuration
}

\begin{abstract}
In this paper, we are proposing a detection scheme known as spectral direct detection technique implemented with Fiber Bragg Grating (FBG) act as encoder/decoder. This FBG based is used to encode and decode the spectral amplitude coding namely modified double weight (MDW) code in Optical Code Division Multiple Access (OCDMA). This code is used due to its flexibility where its weight can be any even number that greater than two. Moreover, it can maintain the cross-correlation parameter equal to one. The performance of spectral direct detection technique against AND-subtraction technique which is both implemented with FBG based encoder/decoder is compared via simulation in downstream and upstream access network at point to multipoint (P2MP) configuration. The simulation will be carried out using OptiSystem version 6.0 and the performance is characterized through bit error rate (BER) and power received at various bit rate.
\end{abstract}

